\title{
The cnidarian parasite Ceratonova shasta utilizes inherited and recruited venom-like compounds during infection
}

\author{
Benjamin Americus ${ }^{1}$, Nicole Hams ${ }^{1}$, Anna Klompen ${ }^{2}$, Gema Alama-Bermejo ${ }^{1,3}$, Tamar Lotan ${ }^{4}$, Jerri L \\ Bartholomew $^{1}$, Stephen D Atkinson ${ }^{\text {Corresp. } 1}$ \\ ${ }^{1}$ Department of Microbiology, Oregon State University, Corvallis, Oregon, United States of America \\ Department of Ecology and Evolutionary Biology, The University of Kansas, Lawrence, Kansas, United States of America \\ 3 Laboratory of Fish Protistology, Institute of Parasitology, Czech Academy of Sciences, České Budějovice, Czech Republic \\ 4 Marine Biology Department, The Leon H. Charney School of Marine Sciences, University of Haifa, Haifa, Israel \\ Corresponding Author: Stephen D Atkinson \\ Email address: atkinsos@oregonstate.edu
}

\section{Background}

Cnidarians are the most ancient venomous organisms. They store a cocktail of venom proteins inside unique stinging organelles called nematocysts. When a cnidarian encounters chemical and physical cues from a potential threat or prey animal, the nematocyst is triggered and fires a harpoon-like tubule to penetrate and inject venom into the prey. Nematocysts are present in all Cnidaria, including the morphologically simple Myxozoa, which are a speciose group of microscopic, spore-forming, obligate parasites of fish and invertebrates. Rather than predation or defense, myxozoans use nematocysts for adhesion to hosts, but the involvement of venom in this process is poorly understood. Recent work shows some myxozoans have a reduced repertoire of venom-like compounds (VLCs) relative to free-living cnidarians, however the function of these proteins is not known.

\section{Methods}

We searched for VLCs in the nematocyst proteome and a time-series infection transcriptome of Ceratonova shasta, a myxozoan parasite of salmonid fish. We used four parallel approaches to detect VLCS: BLAST and HMMER searches to preexisting cnidarian venom datasets, the machine learning tool ToxClassifier, and structural modeling of nematocyst proteomes. Sequences that scored positive by at least three methods were considered VLCs. We then mapped their time-series expressions in the fish host and analyzed their phylogenetic relatedness to sequences from other venomous animals.

\section{Results}

We identified 8 VLCs, all of which have closely related sequences in other myxozoan datasets, suggesting a conserved venom profile across Myxozoa, and an overall reduction in venom diversity relative to freeliving cnidarians. Expression of the VLCs over the three-week fish infection varied considerably: three sequences were most expressed at one day post-exposure in the fish's gills; whereas expression of the other five VLCs peaked at 21 days post-exposure in the intestines, coinciding with the formation of mature parasite spores with nematocysts. Expression of VLC genes early in infection, prior to the development of nematocysts, suggests venoms in $C$. shasta have been repurposed to facilitate parasite invasion and proliferation within the host. Molecular phylogenetics suggested some VLCs were inherited from a cnidarian ancestor, whereas others were more closely related to sequences from venomous nonCnidarian organisms and thus may have gained qualities of venom components via convergent evolution. Peer) reviewing PDF | (2021:07:63176:1:1:NEW 15 Oct 2021) 
The presence of VLCs and their differential expression during parasite infection enrich the concept of what functions a "venom" can have and represent targets for designing therapeutics against myxozoan infections. 
1 The cnidarian parasite Ceratonova shasta utilizes inherited and recruited venom-like compounds

2 during infection.

4 Benjamin Americus ${ }^{1}$, Nicole Hams ${ }^{1}$, Anna M. L. Klompen², Gema Alama-Bermejo ${ }^{3}$, Tamar

5 Lotan $^{4}$, Jerri L. Bartholomew ${ }^{1}$, Stephen D. Atkinson ${ }^{1}$

6

$7 \quad{ }^{1}$ Department of Microbiology, Oregon State University, Corvallis, OR, USA

8 2Department of Ecology and Evolutionary Biology, The University of Kansas, Lawrence, KS, 9 USA

$10{ }^{3}$ Laboratory of Fish Protistology, Institute of Parasitology, České Budějovice, Czech Republic $11{ }^{4}$ Marine Biology Department, The Leon H. Charney School of Marine Sciences, University of 12 Haifa, Israel

Corresponding Author:

15 Stephen D. Atkinson ${ }^{1}$

16 Nash Hall 226, OSU, Corvallis, OR 97331

17 Email address Stephen.Atkinson@oregonstate.edu

\section{Abstract}

Background

Cnidarians are the most ancient venomous organisms. They store a cocktail of venom proteins inside unique stinging organelles called nematocysts. When a cnidarian encounters chemical and physical cues from a potential threat or prey animal, the nematocyst is triggered and fires a harpoon-like tubule to penetrate and inject venom into the prey. Nematocysts are present in all Cnidaria, including the morphologically simple Myxozoa, which are a speciose group of microscopic, spore-forming, obligate parasites of fish and invertebrates. Rather than predation or defense, myxozoans use nematocysts for adhesion to hosts, but the involvement of venom in this process is poorly understood. Recent work shows some myxozoans have a reduced repertoire of venom-like compounds (VLCs) relative to free-living cnidarians, however the function of these proteins is not known.

Methods Ceratonova shasta, a myxozoan parasite of salmonid fish. We used four parallel approaches to detect VLCs: BLAST and HMMER searches to preexisting cnidarian venom datasets, the machine learning tool ToxClassifier, and structural modeling of nematocyst proteomes. Sequences that scored positive by at least three methods were considered VLCs. We then mapped their time-series expressions in the fish host and analyzed their phylogenetic relatedness to sequences from other venomous animals. 
41

42

43

44

45

46

47

48

49

50

51

52

53

54

55

56

57

58

59

60

61

62

63

64

65

66

67

68

69

70

71

72

73

74

75

76

77

78

79

80

Results

We identified 8 VLCs, all of which have closely related sequences in other myxozoan datasets, suggesting a conserved venom profile across Myxozoa, and an overall reduction in venom diversity relative to free-living cnidarians. Expression of the VLCs over the three-week fish infection varied considerably: three sequences were most expressed at one day post-exposure in the fish's gills; whereas expression of the other five VLCs peaked at 21 days post-exposure in the intestines, coinciding with the formation of mature parasite spores with nematocysts. Expression of VLC genes early in infection, prior to the development of nematocysts, suggests venoms in $C$. shasta have been repurposed to facilitate parasite invasion and proliferation within the host. Molecular phylogenetics suggested some VLCs were inherited from a cnidarian ancestor, whereas others were more closely related to sequences from venomous non-Cnidarian organisms and thus may have gained qualities of venom components via convergent evolution. The presence of VLCs and their differential expression during parasite infection enrich the concept of what functions a "venom" can have and represent targets for designing therapeutics against myxozoan infections.

\section{Introduction}

Cnidarians are the earliest diverging extant venomous animals (Jouiaei et al., 2015). This ancient and taxonomically diverse phylum of aquatic invertebrates is characterized by a unique organelle, the nematocyst. Inside each nematocyst is a coiled and eversible tubule, which can be discharged to deliver a venomous sting (Beckman \& Özbek, 2012). Venom is a cocktail of toxic compounds, stored inside the unfired inverted tubule and nematocyst (Lotan, 1995). When a cnidarian encounters chemical and physical cues, the nematocyst is triggered and the tubule everts, injecting the venom into the host or the immediate environment. In Cnidaria, most venom constituents are proteins, broadly comprising enzymes, pore-forming toxins, and neurotoxins (Jouiaei et al., 2015; Rachamim et al., 2015; Podobnik \& Anderluh, 2017; D’Ambra \& Lauritano, 2020). These compounds are useful in immobilizing and digesting prey and deterring predators (Orts et al., 2013). Note that for the many taxa where functional studies have not been undertaken, candidate venoms can be inferred from sequence or structural homology; we refer to these here as venom-like compounds (VLCs).

Nematocyst structure and venom composition vary among the three main clades of Cnidaria: Anthozoa and Medusozoa, which are predominantly free-living, and Endocnidozoa, which contains the enigmatic and parasitic Myxozoa (Rachamim et al., 2015; Kayal et al., 2018, Americus et al., 2020). Myxozoans require two hosts and alternate between vertebrate-infective actinospores and invertebrate-infective myxospores (Okamura et al., 2015). Anthozoa (sea anemones, corals, etc.) and Medusozoa (medusa-bearing species) have a wide variety of nematocyst morphotypes both within and between species (Fautin 2009). The few myxozoan 
81 nematocysts described have tubules with uniform thickness along their lengths, lack spines 82 (Uspenskaya, 1982; Östman, 2000; Ben-David et al., 2016) and have sealed tips (Ben-David et 83 al., 2016; Piriatinskiy et al., 2017; Americus et al., 2020). These are morphologically similar to 84 the atrichous isorhiza-type nematocysts of medusozoans (Cannon \& Wagner 2003; Americus et 85 al., 2020). Rather than being employed for predation or defense, myxozoans use nematocysts for 86 87

When a myxozoan actinospore encounters physical and chemical cues produced by a potential host, the tubule everts, penetrating the host epithelium and anchoring the parasite (Kallert et al., 2007). In some "freshwater" clade Myxobolus spp. the tubule then contracts, pulling the apical end of the spore in contact with the host. Some Myxobolus spp. also discharge nematocyst contents through lateral pores in the tubule, though this is notably absent in the model "marine" clade myxozoan, Ceratonova shasta (Ben-David et al., 2016). Once attached, a multinucleate/multicellular sporoplasm migrates out of the spore and into the host, beginning the infection (Bjork \& Bartholomew 2010; Kallert et al., 2007).

Myxozoans may have lost the venom proteins of their free-living relatives in a genome reduction with the shift to parasitism (Piriatinskiy et al., 2017). This is evidenced by simplifications of morphology and function - primarily for attachment to a host (Kallert et al., 2007). But these reductions differ among myxozoan taxa, for example a transcriptome analysis of Myxobolus pendula cysts in fish identified 49 VLCs (Foox et al., 2015), whereas searches for VLCs in the C. shasta nematocyst proteome (Piriatinskiy et al., 2017) identified only a single venom-like domain in a protein of unknown function, which also supports the hypothesis that myxozoan nematocysts do not contain venoms. There is, however, some evidence for myxozoans having VLCs localized outside nematocysts (Hartigan et al., 2021). This is known from free-living cnidarians, where venoms may be secreted from glands in the ectoderm and pharynx for prey immobilization and digestion (Moran et al., 2012, 2013; Zhang et al., 2003).

Hartigan et al. (2021) recently identified VLCs in transcriptomes and proteomes from the myxozoans Buddenbrockia plumatellae, Myxobilatus gasterostei and Sphaerospora elegans, the semi-parasitic endocnidozoan Polypodium hydriforme, and the free-living medusozoan Calvadosia cruxmelitensis. The myxozoans had only $1 / 3$ the venom diversity relative to the freeliving species, whereas $P$. hydriforme had $2 / 3$ the venom diversity of the free-living species, which is consistent with its intermediate phylogenetic position between free-living cnidarians and Myxozoa (Kayal et al., 2018). Some VLCs in the endocnidozoan datasets appear inherited from free-living cnidarians, with phylogenies correlating with established taxonomy. Other VLCs were more closely related to those from venomous animals outside of Cnidaria, suggesting convergent evolution and a recruitment of venoms for other physiological roles. 
120 In this study, we continue the search for VLCs in myxozoans. We reexamine the proteome of $C$. 121 shasta nematocysts (Piriatinskiy et al., 2017) and a time-series of infection transcriptome (Barrett 122 \& Bartholomew, 2021) to identify candidate VLCs and infer their functions. We hypothesize that 123 C. shasta has retained a venom arsenal from a cnidarian ancestor and predict that adaptation to a 124 parasitic lifestyle has led to reduction in venom diversity relative to extant free-living species. 125 We further hypothesize that the C. shasta VLCs are highly dissimilar from those of extant free126 living Cnidaria but will have retained recognizable functional "venom" domains, as is the case in 127 myxozoan ribosomal (Evans et al., 2010) and mitochondrial genes (Takeuchi et al., 2015).

128

129

Materials \& Methods

130

131

We used four parallel approaches to identify candidate sequences of venom-like compounds 132

133 (VLCs): 1) structural phylogenetics, 2) BLAST, 3) HMMER, and 4) ToxClassifier (Fig. 1). We considered sequences that were positive by at least three methods to be putative VLCs. In the case of multiple VLCs from a single Trinity read cluster, we selected the longest sequence for downstream analysis. Sequences in the infection transcriptome and their identical translations in the nematocyst proteome were considered to be single VLCs.

137

138

Functional prediction by structural modeling

139

140

To predict the tertiary structure of the $C$. shasta nematocyst peptides (Piriatinskiy et al., 2017), we submitted them to the Phyre2 server (Kelley et al., 2015) with the Normal modelling mode. Protein function was assigned based on homology to known structures in the Protein Data Bank (PBD) or families in the Structural Classification of Proteins database (accessed March 2019). Results were parsed by the presence of structurally similar sequences whose functions contained keywords (protease, inhibitor, toxin, venom). We submit structures to the Dali Protein Structure Comparison Server all-against-all structure comparison tool (Holm \& Laakso 2016) for tree

147 building (Supplemental File 2). We did not perform this analysis on the infection transcriptome 148 (42,542 transcripts) due to the uncertainties introduced in peptide prediction, and limitations of 149 manual analysis.

150

\section{Homology search}

152

153

We manually curated a database of venom and toxin proteins and transcripts from publicly

154

155 available venom transcriptomes and proteomes: box jellyfish Alatina alata (Ames et al., 2016), pore-forming proteins from several free-living cnidaria (Podobnik \& Anderluh 2017), putative toxin transcripts from the acrorhagi of Anthopleura elgantissima sea anemones (Macrander et al., 2015), venom transcripts from the tentacles, mesentery, and column of Anemonia sulcata, Heteractis crispa, and Megalactis griffithsi anemones (Macrander et al., 2016), venom protein sequences from the box jellyfish Chironex fleckeri (Brinkman et al., 2012), protein contents of 
160 the nematocysts of the sea anemone Nematostella vectensis (Moran et al., 2013), and the UniProt

161

162

163

164

165

166

167

168

169

170

171

172

173

174

175

176

177

178

179

180

181

182

183

184

185

186

187

188

189

190

191

192

193

194

195

196

197

198

manually-annotated animal venom and toxin protein database (downloaded January, 2019). Our custom database consisted of 31,798 transcripts and 7,129 proteins. We screened the $C$. shasta nematocyst proteome and infection transcriptome against this database using BLAST searches (e-value cutoff 1e-5). In parallel, we screened the same $C$. shasta sequences against the cnidarian venom-like HMM database from Klompen et al. (2020), with a less stringent e-value cutoff (1e3) to allow comparison with similar work by Klompen et al. (2020) on Cerianthid transcriptomes.

\section{ToxClassifier}

We input the nematocyst proteome and translated infection transcriptome into the web-based version of the machine learning tool ToxClassifier (Gacesa et al., 2016). We considered sequences deemed as either "toxin" or "dubious" by one or more classifiers as positive hits for selecting VLCs.

Phylogenetics of putative venom-like compounds

We constructed phylogenetic trees of the putative VLCs identified by at least three screening methods. We used BLASTP to identify and download homologous sequences from GenBank (queried November 2020). To test our hypothesis of venom inheritance from a cnidarian ancestor, we included sequences from Polypodium hydriforme (the non-myxozoan member of Endocnidozoa), which is an evolutionary intermediate between Myxozoa and free-living Cnidaria, and which has nematocysts that are structurally similar to the venomous holotrichous isorhiza of free-living cnidarians (Ibragimov \& Raikova, 2004). We also included sequences from Medusozoa (sister to Endocnidozoa), more distantly related anthozoans, and rooted the tree using homolgous sequences from Porifera. Sequences were aligned with MUSCLE (Edgar, 2004), then trimmed, and the best alignment models selected using MEGA-X (version 10.2.5, Kumar et al., 2018). We constructed maximum likelihood trees with IQ-TREE (Version 2.1.2, Minh et al., 2020) using 1000 bootstrap replicates. We used FigTree (version 1.4.4, http://tree.bio.ed.ac.uk/// to visualize all trees and we included ultrafast bootstrap support values $>50$. We compared the topology of our single-gene trees to phylogenomic trees constructed from whole-transcriptome comparison (Kayal et al., 2018; Chang et al., 2015), to ascertain whether the gene was inherited from a common medusozoan ancestor or acquired some other way. The untrimmed transcripts for each tree were input into the NCBI conserved domains search tool (Marchler-Bauer et al., 2015) for domain visualization.

\section{Analysis of time-series expression during infection in fish}


199 The time-series infection data were obtained from Barrett \& Bartholomew (2021), (NCBI 200 BioProject PRJNA694439), from susceptible rainbow trout that were exposed to C. shasta 201 genotype IIR for $24 \mathrm{~h}$, then held for $21 \mathrm{~d}$. Gills were sampled at $1 \mathrm{~d}$ post-exposure (dpe), and 202 intestines were sampled at 7, 14, and 21 dpe. Fish exposures, RNA-extraction and sequencing are 203 described in detail in Barrett \& Bartholomew (2021). RNA-Seq data were generated using an 204 Illumina HiSeq 3000 with 100-bp single-end runs. We used BBDuk (version 38.11, 205 https://sourceforge.net/projects/bbmap/) to remove reads with $>20$ bases with PHRED score $<20$, 206 reads containing homopolymers $>50$ bases, and reads with $>=20$ bases overlapping with Illumina 207 Adapters. The remaining "cleaned" reads from all timepoints were pooled, host-filtered, and assembled into a reference transcriptome using a published pipeline (Alama-Bermejo et al., 2020). A newer version of the host genome was used for mapping during host contamination filtration (NCBI Assembly GCF_002163495.1). We retained reads that mapped to the $C$. shasta genome, and neither $C$. shasta nor host genomes. We used Transdecoder (version 5.5.0, Haas et al., 2013), with default settings and the-single_best_orf option to generate amino acid sequences

214 (Piriatinskiy et al., 2017) were annotated using HMMER (version 3.3, hmmer.org) to compare

215 216

217 To generate a read count matrix for transcripts at each timepoint, we used Salmon (version 218 0.10.0, Patro et al., 2017) to pseudo-align reads from each timepoint to the time-series 219 transcriptome. We input read counts into DESeq2 (version 3.12, Love et al., 2014) using

220

221

222

223

224

225

226

227

228

229

230

231

232

233

234

235

236

237

238 tximport (Soneson et al., 2015). To correct for parasite replication within the host, we used the estimateSizeFactors function in DESeq2 to normalize transcript counts based on two myxozoan single-copy genes: glyceraldehyde-3-phosphate dehydrogenase and eukaryotic translation elongation factor 2 (Kosakyan et al., 2019). We analyzed expression in terms of read count per gene rather per isoform to better distinguish functional roles within the host. Read counts underwent variance-stabilizing transformation prior to downstream analysis. To distinguish coexpressed genes, we input normalized read counts into the Short Time-series Expression Miner (STEM; version 1.3.13, Ernst \& Bar-Joseph 2006) with default settings and without normalization and " 0 " as an initial datapoint to reflect the absence of parasite genes in the host prior to infection.

\section{Results}

\section{Identification of candidate venom-like compounds}

The overlap of all venom screening methods is displayed in Fig. 2. From the 114-peptide nematocyst proteome, 110 had structurally similar sequences in PBD or the Structural Classification of Proteins database, 48 of which had annotations containing target keywords (protease, inhibitor, toxin, venom). From this reduced set, 28 sequences contained complete, 
239 high-confidence ( $>95 \%$ Phyre 2 confidence) structural domains and were retained for structural

240 phylogenetics (Supplemental File 1). From the whole proteome, 26 sequences had BLAST

241 homologs in the manually constructed venom database, 4 sequences had hits to HMMs, and 8

242 sequences were positive hits by ToxClassifier (Supplemental File 2). The de-novo assembled

243 infection transcriptome consisted of 42,543 transcripts with an N50 of $1120 \mathrm{bp}$. The

244 transcriptomic dataset we searched was $1 / 2-1 / 5$ the size of those used by prior myxozoan venom

245 studies (Foox et al., 2016, Hartigan et al., 2021) despite comparable sequencing depths. We used

$246100 \mathrm{bp}$ single-end reads (whereas prior studies used paired-end), and we applied an additional

247 level of contig filtering by annotation. 884 sequences had BLAST homologs in our venom

248 database, 71 sequences had venom-like domains predicted by HMMs, and 468 sequences were

249 deemed positive hits by ToxClassifier (Supplemental File 2). HMMs with homologous

250 sequences from each dataset are displayed in Fig. 3.

251

252

253

We identified two unique Kunitz-type protease inhibitor-like proteins based on similarity to

254

255

256

257

258

259

260

261

262

263

264

265

266

267

268

269

270

271

272

273

274

275

276

277

278

sequences in Genbank. We refer to these as Kunitz-type protease inhibitor-like transcript 1 ), and Kunitz-type protease inhibitor-like transcript 2). In two cases, the C-type lectin-like protein and the peptidase inhibitor 16-like protein, identical, shorter sequences were recovered from the infection transcriptome. We selected the longer sequences from the nematocyst proteome for downstream analysis.

Altogether, 8 candidate VLCs passed our screening methods (Table 1). Those from the nematocyst proteome that were positive by at least three methods included a lactadherin-like protein, a peptidase inhibitor-16-like protein, and a C-type lectin-like protein. The infection transcriptome contained two metallopeptidase-like transcripts (astacin and reprolysin-like), two Kunitz-type protease inhibitor-like transcripts, a hyaluronidase-like transcript, and the same peptidase-inhibitor 16-like transcript and C-type lectin-like transcript found in the nematocyst proteome.

\section{Phylogenetic analysis of VLCs}

We found homologs to the candidate $C$. shasta VLCs in all major classes of Cnidaria, including Polypodiozoa, Myxosporea, Malacosporea, Anthozoa and Medusozoa. We identified $P$. hydriforme homologs to 7 of our 8 C. shasta VLCs. For two, the reprolysin-like and peptidase inhibitor 16-like protein, we identified similar sequences in the malacosporean Tetracapsuloides bryosalmonae in transcriptomes from its invertebrate host but not fish host. For the astacin-like and Kunitz-type protease inhibitor-like transcript 2 transcripts, we found similar sequences in BLAST searches from venomous organisms outside Cnidaria (predominantly from class Arachnida). We included these sequences in the alignments and trees. We also included homologous sequence from the sponge Amphimedon queenslandica for all VLCs except the hyaluronidase-like transcript, for which a sequence was not available.

Peer) reviewing PDF | (2021:07:63176:1:1:NEW 15 Oct 2021) 
279

280 Maximum likelihood trees and corresponding alignments are displayed in Fig. 4 and

281

282

283

284

285

286

287

288

289

290

291

292

293

294

295

296

297

298

299

300

301

302

303

304

305

306

307

308

309

310

311

312

313

314

315

316

317

318

Supplemental File 3. Several VLCs appear to be incomplete or are missing functional domains contained in related sequences. It is unknown whether this is an artifact of de-novo transcriptome assembly or is biological reality. For three VLCs - the lactadherin-like protein, the C-type lectinlike protein, and hyaluronidase-like transcript - the topology of phylogenetic trees agree with taxonomy established via phylogenomic trees (Kayal et al., 2018; Chang et al., 2015). Five VLCs did not have topologies in agreement with taxonomy. These included the Kunitz-type protease inhibitor-like transcripts, the peptidase inhibitor 16-like protein, and both metallopeptidase-like transcripts.

Time-series expression of VLCS

More VLC gene transcripts in the infection transcriptome were expressed later in infection (42\% at $1 \mathrm{dpe}, 46 \%$ at $7 \mathrm{dpe}, 85 \%$ at $14 \mathrm{dpe}$, and $>99 \%$ at $21 \mathrm{dpe}$. The largest single gene cluster identified by STEM, peaked at 1 dpe in the gills and decreased at later timepoints in the intestine, and included $30 \%$ of all genes. The nematocyst proteome homologs were expressed similarly (56\% at $1 \mathrm{dpe}, 60 \%$ at $7 \mathrm{dpe}, 99 \%$ at $14 \mathrm{dpe}$, and $100 \%$ at $21 \mathrm{dpe}$ ). The largest STEM cluster included $27 \%$ of genes with expression peaking at $1 \mathrm{dpe}$.

The three VLCs identified in the $C$. shasta nematocyst proteome matched highly similar sequences (tblastn e-value $=0$ ) in the infection transcriptome. Of the 8 candidates, three were expressed at 1 dpe (in gills) (Fig. 5). These include the C-type lectin-like protein, the hyaluronidase-like transcript, and the Kunitz-type protease inhibitor-like transcript 1 . The 5 others had expression beginning at 14 or 21 dpe and were assigned to two other STEM clusters. These include the peptidase inhibitor 16-like protein, the lactadherin-like protein, the Kunitztype protease inhibitor-like transcript 2 and the reprolysin and astacin metallopeptidase-like transcripts.

\section{Discussion}

Our multifaceted approach yielded 8 VLCs in C. shasta, 7 of which have been identified in other myxozoan species (Foox et al., 2015; Hartigan et al., 2021). These include C-type lectin-like proteins, metallopeptidases-like transcripts, peptidase-inhibitor-like proteins and Kunitz-type protease inhibitor-like transcripts. We found hyaluronidase-like transcripts for the first time in a myxozoan. Below, we discuss the individual C. shasta VLCs organized by postulated evolutionary origin; low bootstrap values on many of our VLC phylogenies limit the strength of support for these hypotheses.

\section{"Inherited" VLCs}


319

320

321

322

323

324

325

326

327

328

329

330

331

332

333

334

335

336

337

338

339

340

341

342

343

344

345

346

347

348

349

350

351

352

353

354

355

356

357

358
Phylogenetic trees of three $C$. shasta VLCs have topologies that parallel taxonomy, and thus we consider this as support for the hypothesis that some VLCs are inherited from a common cnidarian ancestor.

\section{Lactadherin-like protein}

Lactadherins are anticoagulants that compete with host blood-clotting factors (Shi \& Gilbert, 2003). They are present in venom from snakes (Ching et al., 2012) and free-living cnidarians (Yang et al., 2014; Voolstra et al., 2017). The lactadherin-like sequence we identified had expression coinciding with the appearance of mature spores in histology at $21 \mathrm{dpe}$ (Barrett \& Bartholomew, 2021). The FA58C domains contained within this sequence is related to blood coagulation and has been noted in snake datasets (Ching et al., 2012; Rokyta et al., 2013; Junqueira-de-Azevedo et al., 2016). Potentially, lactadherin is translated late in infection to be packaged into mature nematocysts. While $C$. shasta tubules appear sealed, these venom peptides may be delivered in a mucus coating (Ramírez-Carreto et al., 2019) on the tubules, to suppress host blood clotting during initial attachment of the parasites.

\section{C-type lectin-like protein}

C-type lectins disrupt hemostasis and inflammation and are widely distributed in Cnidaria and other venomous animals (Morita 2005). The C-type-lectin we identified is homologous to those recently identified in sea anemone transcriptomes (Klompen et al., 2020). In our infection transcriptome, C-type lectin expression peaked at 1 dpe in the gills. At 1 dpe, C. shasta is in an "amoeboid migration" phase, traveling via blood vessels from the site of entry to the intestine (Alama-Bermejo, Holzer \& Bartholomew 2019; Bjork \& Bartholomew 2010). At later timepoints, coinciding with $C$. shasta embedding in the intestine, this transcript had lower expression. Thus, we hypothesize that C-type lectin may help C. shasta evade the host innate immune response during initial migration (Loukas et al., 1999), and in the nematocysts, may serve a secondary, more typical venom function by disrupting host coagulation during attachment.

\section{Hyaluronidase-like transcript}

Hyaluronidases are regarded as "spreading factors" as they degrade hyaluronan to disrupt extracellular membranes, allowing the spread of toxins to local tissues (Tu \& Hendon, 1983). They are present in the venom of many cnidarian taxa, including jellyfish (Lee et al., 2011), and sea anemones (Domínguez-Pérez et al., 2018). In C. shasta, we observed the hyaluronidase-like transcript was highly expressed at 1 dpe in the gills, and we propose that it may disrupt gill tissue to enable sporoplasm invasion. At $7 \mathrm{dpe}$, though expression was lower, hyaluronidase may

PeerJ reviewing PDF | (2021:07:63176:1:1:NEW 15 Oct 2021) 
359

360

361

362

363

364

365

366

367

368

369

370

371

372

373

374

375

376

377

378

379

380

381

382

383

384

385

386

387

388

389

390

391

392

393

394

395

396

397

398

facilitate the amoeboid "blebbing" motility described by Alama-Bermejo et al. (2019), which could facilitate parasite penetration through gaps in host cellular matrices.

"Recruited" VLCs

Phylogenetic trees of the other 5 VLCs do not parallel established cnidarian topologies, despite all having sequence motifs characteristic of known venom components. We hypothesize that these compounds took on venom-like qualities via independent origins and convergent evolution, similar to what has been proposed with serine proteases inhibitors (Eszterbauer et al., 2020) and venom components (Hartigan et al., 2021) from other myxozoan taxa. We refer to these candidates as "recruited". Recruitment of proteins for venom is common for venomous taxa including cnidarians (Jaimes-Becerra et al., 2017), platypus, and snakes (Whittington et al., 2008; Fry et al., 2009).

\section{Peptidase Inhibitor16-like sequences}

Peptidase inhibitor 16 (PI16) belongs to the CAP superfamily of proteins, and has been identified in venom proteomes (e.g. cone snails, Leonardi et al., 2012) and transcriptomes (e.g. scorpions, Cid-Uribe et al., 2018). Whereas its role in venoms is still unknown, in humans PI16 expression has been connected to conditions including heart disease (Frost et al., 2007), cancer (Wang et al., 2020), and inflammation (Hazell et al., 2016). PI16 prevents the proteolytic activation of chemerin, a protein involved in recruiting macrophages to sites of inflammation (Regn et al., 2016). A PI16-like protein was discovered in the original description of the $C$. shasta nematocyst proteome (Piriatinskiy et al., 2017), and we recovered it again using our methods. The transcript encoding this protein was expressed at 14 and $21 \mathrm{dpe}$, while the parasite is proliferating. While the role of these proteins in free-living Cnidaria remains unknown, we hypothesize that in $C$. shasta infections, peptidase inhibitors are involved in host immune silencing to enable parasite replication, a phenomenon discussed in Barrett \& Bartholomew, (2021).

\section{Metallopeptidase-like sequences}

Metallopeptidases are proteolytic enzymes that utilize metals to cleave polypeptide bonds. They have various functions in organism development and are found in venom from snakes (Bjarnason \& Fox, 1995), scorpions (Cid-Uribe et al., 2018) and cnidarians (Moran et al., 2013). In C. shasta, proteases are an essential aspect of virulence and vary greatly between genotypes (Alama-Bermejo et al., 2020). The two metallopeptidase VLCs we identified have different annotations and expression profiles and should be considered independently. Reprolysins are a subfamily of metalloproteinases known from snake venoms (Bjarnason \& Fox, 1995). The reprolysin-like transcript was expressed at all timepoints and in both gill and gut tissue, where it may cleave host tissue for movement and proliferation. Astacins are membrane-secreted 
399

400

401

402

403

404

405

406

407

408

409

410

411

412

413

414

415

416

417

418

419

420

421

422

423

424

425

426

427

428

429

430

431

432

433

434

435

436

437

metalloendopeptidase in sea anemone venom. The astacin-like transcript was not expressed until 21 dpe. Like the lactadherin-like VLC, this late-expressed gene may be translated and packaged in nematocysts of mature myxospores and have a role in infecting the invertebrate host.

\section{Kunitz-type protease inhibitor-like transcripts}

Kunitz-type protease inhibitors are a family of protease inhibitors widespread in venomous taxa (Yang et al., 2014). In sea anemones, Kunitz-type protease inhibitor proteins in nematocysts and mucus defend against proteolysis by predators and prey (Sinstova et al., 2018). Some parasites use Kunitz-type protease inhibitors to interfere with host immune response (Ranasinghe et al., 2015; Smith et al., 2020). We identified two Kunitz-type inhibitor proteins that have similar annotations but distinct expression profiles. Transcript 1 was heavily expressed at 1 dpe in the gills, and less at 7, 14 and 21 dpe in the intestines, whereas Transcript 2 was not expressed until 14 dpe. Asynchronous expression of protease inhibitors was likewise observed in M. cerebralis infections in Rainbow trout (Eszterbauer et al., 2021). Like these proteins in M. cerebralis, Kunitz-type protease inhibitors in C. shasta may have several roles in evading host immunity.

\section{Sequences containing the ShK domain}

Stichodacyla helianthus K -like (ShK) domain are small proteins that interfere with potassium channels. They are ubiquitous in sea anemone venom (Sachkova et al., 2020) and have structural orthologs in venomous organisms outside Cnidaria (Gerdol et al., 2019). Both of our datasets contained sequences with ShK-like domains detected by HMMER searches of the pfam database and venom HMMs from Klompen et al. (2020). While we are not prepared to ascribe these sequences as VLCs, they are notable targets for future investigation, as Shk domains also function in cnidarian nervous systems (Sachkova et al., 2020), which have not yet been observed in myxozoans.

\section{Conclusions}

We used a multi-faceted approach to identify putative VLCs in C. shasta proteomic and transcriptomic datasets. Relative to other cnidarians, VLCs in C. shasta are remarkably scarce: Similar approaches in free-living cnidarians have yielded $\sim 100-600$ candidate genes per species (Brinkman et al., 2015; Macrander et al., 2015; Macrander et al., 2016; Ames et al., 2016; Klompen et al., 2020). However, we identified only 8, which are a subset of the 49 "toxins" identified in Myxobolus pendula (Foox et al., 2015), 124 in B. pumatellae and 96 from mixed myxosporean datasets (Hartigan et al., 2021). The $\sim 90 \%$ reduction in VLC diversity in C. shasta relative to venoms in free-living cnidaria parallels the $\sim 95 \%$ reduction in genome size in Myxozoa relative to free-living cnidarians (Chang et al., 2015). Reduced VLC diversity in $C$. 
438 shasta may be an artefact of our different sequencing and assembly parameters, and more-

439 extensive manual screening.

440

441 Our novel time-series data suggest venom-like compounds function during different phases of

442 the C. shasta infection of the fish host, corresponding with invasion, migration and replication of

443 the parasite. Thus these VLCs do not fit the 'predation and defense' narrative of venom function

444 in free-living cnidarians, instead, show that "venom" may have a diverse utility in parasitic

445 organisms. The multi-faceted approach we used in this study should provide a means of

446 identifying and describing venom components and other highly divergent proteins in Myxozoa.

447 In addition, future studies could fluorescently label putative VLCs to determine their localization

448 in myxosporean nematocysts, developmental stages, and mature spores to characterize atypical

449 venom functions. Searches for VLCs in 'omics datasets from myxozoan infections in

450 corresponding invertebrate hosts may reveal multiple functions of the same compounds in

451 alternate hosts of a complex life cycle. Given that we found a greater number of sequences

452 similar to our VLCs in invertebrate infections of T. bryosalmonae, it may be that this venom

453 reduction is less pronounced in the vertebrate-infective stages of myxozoans. Data that improve

454 our understanding of the role of these compounds at the host-parasite interface during invasion

455 and proliferation will simultaneously enrich the concept of what functions a "venom" can have

456 and guide potential treatments for myxozoan infections.

457

458

459

460

461

462

463

464

465

466

467

468

469

470

471

472

473

474

475

476

477

478

\section{Acknowledgements}

Bioinformatic analyses were performed on the computational infrastructure of the Oregon State University Center for Quantitative Life Sciences.

\section{References}

Alama-Bermejo G, Holzer AS, Bartholomew JL. 2019. Myxozoan Adhesion and Virulence: Ceratonova shasta on the Move. Microorganisms 7:397. DOI: 10.3390/microorganisms7100397.

Alama-Bermejo G, Meyer E, Atkinson SD, Holzer AS, Wiśniewska MM, Kolísko M, Bartholomew JL. 2020. Transcriptome-Wide Comparisons and Virulence Gene Polymorphisms of Host-Associated Genotypes of the Cnidarian Parasite Ceratonova shasta in Salmonids. Genome Biology and Evolution 12:1258-1276.

Americus B, Lotan T, Bartholomew JL, Atkinson SD. 2020. A comparison of the structure and function of nematocysts in free-living and parasitic cnidarians (Myxozoa). International Journal for Parasitology 50:763-769.

Barrett D, Bartholomew JL. 2021. A Tale of Two Fish: Comparative Transcriptomics of Resistant and Susceptible Steelhead Following Exposure to Ceratonova Shasta Highlights Differences in Parasite Recognition. PLOS ONE 16(2): e0234837.

Beckmann A, Özbek S. 2012. The nematocyst: a molecular map of the cnidarian stinging organelle. The International Journal of Developmental Biology 56:577-582. 
479

480

481

482

483

484

485

486

487

488

489

490

491

492

493

494

495

496

497

498

499

500

501

502

503

504

505

506

507

508

509

510

511

512

513

514

515

516

517

518

519

520

521

522

523

524

Ben-David J, Atkinson SD, Pollak Y, Yossifon G, Shavit U, Bartholomew JL, Lotan T. 2016. Myxozoan polar tubules display structural and functional variation. Parasites \& Vectors 9:549.

Bjarnason JB, Fox JW. 1995. Snake venom metalloendopeptidases: reprolysins. Methods in Eenzymology 248:345-368.

Bjork SJ, Bartholomew JL. 2010. Invasion of Ceratomyxa shasta (Myxozoa) and comparison of migration to the intestine between susceptible and resistant fish hosts. International Journal for Parasitology 40:1087-1095.

Brinkman DL, Aziz A, Loukas A, Potriquet J, Seymour J, Mulvenna J. 2012. Venom proteome of the box jellyfish Chironex fleckeri. PLOS ONE 7:e47866.

Brinkman DL, Jia X, Potriquet J, Kumar D, Dash D, Kvaskoff D, Mulvenna J. 2015. Transcriptome and venom proteome of the box jellyfish Chironex fleckeri. BMC Genomics 16:407.

Cannon Q, Wagner E. 2003. Comparison of Discharge Mechanisms of Cnidarian Cnidae and Myxozoan Polar Capsules. Reviews in Fisheries Science 11:185-219. DOI: 10.1080/10641260390244305.

Chang ES, Neuhof M, Rubinstein ND, Diamant A, Philippe H, Huchon D, Cartwright P. 2015. Genomic insights into the evolutionary origin of Myxozoa within Cnidaria. Proceedings of the National Academy of Sciences 112:14912-14917.

Ching ATC, Paes Leme AF, Zelanis A, Rocha MMT, Furtado M de FD, Silva DA, Trugilho MRO, da Rocha SLG, Perales J, Ho PL, Serrano SMT, Junqueira-de-Azevedo ILM. 2012. Venomics profiling of Thamnodynastes strigatus unveils matrix metalloproteinases and other novel proteins recruited to the toxin arsenal of rear-fanged snakes. Journal of Proteome Research 11:1152-1162.

Cid-Uribe JI, Santibáñez-López CE, Meneses EP, Batista CVF, Jiménez-Vargas JM, Ortiz E, Possani LD. 2018. The diversity of venom components of the scorpion species Paravaejovis schwenkmeyeri (Scorpiones: Vaejovidae) revealed by transcriptome and proteome analyses. Toxicon 151:47-62.

Columbus-Shenkar YY, Sachkova MY, Macrander J, Fridrich A, Modepalli V, Reitzel AM, Sunagar K, Moran Y. 2018. Dynamics of venom composition across a complex life cycle. eLife 7. DOI: 10.7554/eLife.35014.

D'Ambra I, Lauritano C. 2020. A Review of Toxins from Cnidaria. Marine Drugs 18. DOI: 10.3390/md18100507.

Domínguez-Pérez D, Campos A, Alexei Rodríguez A, Turkina MV, Ribeiro T, Osorio H, Vasconcelos V, Antunes A. 2018. Proteomic Analyses of the Unexplored Sea Anemone Bunodactis verrucosa. Marine Drugs 16. DOI: 10.3390/md16020042.

Edgar RC. 2004. MUSCLE: a multiple sequence alignment method with reduced time and space complexity. BMC Bioinformatics 5:113.

Ernst J, Bar-Joseph Z. 2006. STEM: a tool for the analysis of short time series gene expression data. BMC Bioinformatics 7:191.

Eszterbauer E, Sipos D, Kaján GL, Szegő D, Fiala I, Holzer AS, Bartošová-Sojková P. 2020. Genetic Diversity of Serine Protease Inhibitors in Myxozoan (Cnidaria, Myxozoa) Fish Parasites. Microorganisms 8. DOI: 10.3390/microorganisms8101502.

Eszterbauer E, Szegő D, Ursu K, Sipos D, Gellért Á. 2021. Serine protease inhibitors of the whirling disease parasite Myxobolus cerebralis (Cnidaria, Myxozoa): Expression profiling and functional predictions. PLOS ONE 16:e0249266.

Peer] reviewing PDF | (2021:07:63176:1:1:NEW 15 Oct 2021) 
525

526

527

528

529

530

531

532

533

534

535

536

537

538

539

540

541

542

543

544

545

546

547

548

549

550

551

552

553

554

555

556

557

558

559

560

561

562

563

564

565

566

567

568

569

Evans NM, Holder MT, Barbeitos MS, Okamura B, Cartwright P. 2010. The phylogenetic position of Myxozoa: exploring conflicting signals in phylogenomic and ribosomal data sets. Molecular Biology and Evolution 27:2733-2746.

Fautin DG. 2009. Structural diversity, systematics, and evolution of cnidae. Toxicon 54:1054 1064.

Fiala I. 2006. The phylogeny of Myxosporea (Myxozoa) based on small subunit ribosomal RNA gene analysis. International Journal for Parasitology 36:1521-1534.

Foox J, Ringuette M, Desser SS, Siddall ME. 2015. In silico hybridization enables transcriptomic illumination of the nature and evolution of Myxozoa. BMC Genomics 16:840.

Frost RJA, Engelhardt S. 2007. A Secretion Trap Screen in Yeast Identifies Protease Inhibitor 16 as a Novel Antihypertrophic Protein Secreted From the Heart. Circulation 116:1768-1775. DOI: 10.1161/circulationaha.107.696468.

Fry BG, Roelants K, Champagne DE, Scheib H, Tyndall JDA, King GF, Nevalainen TJ, Norman JA, Lewis RJ, Norton RS, Renjifo C, de la Vega RCR. 2009. The toxicogenomic multiverse: convergent recruitment of proteins into animal venoms. Annual review of genomics and human genetics 10:483-511. DOI: 10.1146/annurev.genom.9.081307.164356

Gacesa R, Barlow DJ, Long PF. 2016. Machine learning can differentiate venom toxins from other proteins having non-toxic physiological functions. PeerJ Computer Science 2:e90. DOI: $10.7717 /$ peerj-cs.90.

Gerdol M, Cervelli M, Mariottini P, Oliverio M, Dutertre S, Modica MV. 2019. A Recurrent Motif: Diversity and Evolution of ShKT Domain Containing Proteins in the Vampire Snail. Toxins 11. DOI: 10.3390/toxins 11020106.

Haas BJ, Papanicolaou A, Yassour M, Grabherr M, Blood PD, Bowden J, Couger MB, Eccles D, Li B, Lieber M, MacManes MD, Ott M, Orvis J, Pochet N, Strozzi F, Weeks N, Westerman R, William T, Dewey CN, Henschel R, LeDuc RD, Friedman N, Regev A. 2013. De novo transcript sequence reconstruction from RNA-seq using the Trinity platform for reference generation and analysis. Nature Protocols 8:1494-1512.

Hartigan A, Jaimes-Becerra A, Okamura B, Doonan LB, Ward M, Marques AC, Long PF. 2021. Recruitment of toxin-like proteins with ancestral venom function supports endoparasitic lifestyles of Myxozoa. PeerJ 9:e11208. DOI:

Hazell GGJ, Peachey AMG, Teasdale JE, Sala-Newby GB, Angelini GD, Newby AC, White SJ. 2016. PI16 is a shear stress and inflammation-regulated inhibitor of MMP2. Scientific Reports 6. DOI: 10.1038/srep39553.

Heberle H, Meirelles GV, da Silva FR, Telles GP, Minghim R. 2015. InteractiVenn: a web-based tool for the analysis of sets through Venn diagrams. BMC Bioinformatics 16:169.

Holm L, Laakso LM. 2016. Dali server update. Nucleic Acids Research 44:W351-W355. DOI: 10.1093/nar/gkw357.

Holzer AS, Bartošová-Sojková P, Born-Torrijos A, Lövy A, Hartigan A, Fiala I. 2018. The joint evolution of the Myxozoa and their alternate hosts: A cnidarian recipe for success and vast biodiversity. Molecular Ecology 27:1651-1666.

Ibragimov A, Raikova E. 2004. Nematocysts of Polypodium hydriforme, a cnidarian parasite of acipenseriform fishes. Hydrobiologia 530-531:165-171. DOI: 10.1007/s10750-004-2651-y.

Jaimes-Becerra A, Chung R, Morandini AC, Weston AJ, Padilla G, Gacesa R, Ward M, Long PF, Marques AC. 2017. Comparative proteomics reveals recruitment patterns of some protein families in the venoms of Cnidaria. Toxicon 137:19-26. 
570

571

572

573

574

575

576

577

578

579

580

581

582

583

584

585

586

587

588

589

590

591

592

593

594

595

596

597

598

599

600

601

602

603

604

605

606

607

608

609

610

611

612

613

614
Jouiaei M, Yanagihara AA, Madio B, Nevalainen TJ, Alewood PF, Fry BG. 2015. Ancient Venom Systems: A Review on Cnidaria Toxins. Toxins 7:2251-2271.

Junqueira-de-Azevedo ILM, Campos PF, Ching ATC, Mackessy SP. 2016. Colubrid Venom Composition: An -Omics Perspective. Toxins 8. DOI: 10.3390/toxins8080230.

Kallert DM, Ponader S, Eszterbauer E, El-Matbouli M, Haas W. 2007. Myxozoan transmission via actinospores: new insights into mechanisms and adaptations for host invasion.

Parasitology 134:1741-1750.

Kayal E, Bentlage B, Sabrina Pankey M, Ohdera AH, Medina M, Plachetzki DC, Collins AG, Ryan JF. 2018. Phylogenomics provides a robust topology of the major cnidarian lineages and insights on the origins of key organismal traits. BMC Evolutionary Biology 18. DOI: 10.1186/s12862-018-1142-0.

Kelley LA, Mezulis S, Yates CM, Wass MN, Sternberg MJE. 2015. The Phyre2 web portal for protein modeling, prediction and analysis. Nature Protocols 10:845-858.

Klompen AML, Macrander J, Reitzel AM, Stampar SN. 2020. Transcriptomic Analysis of Four Cerianthid (Cnidaria, Ceriantharia) Venoms. Marine Drugs 18. DOI: 10.3390/md18080413.

Kosakyan A, Alama-Bermejo G, Bartošová-Sojková P, Born-Torrijos A, Šíma R, Nenarokova A, Eszterbauer E, Bartholomew J, Holzer AS. 2019. Selection of suitable reference genes for gene expression studies in myxosporean (Myxozoa, Cnidaria) parasites. Scientific Reports 9:15073.

Kumar S, Stecher G, Li M, Knyaz C, Tamura K. 2018. MEGA X: Molecular Evolutionary Genetics Analysis across Computing Platforms. Molecular Biology and Evolution 35:15471549 .

Lee H, Jung E-S, Kang C, Yoon WD, Kim J-S, Kim E. 2011. Scyphozoan jellyfish venom metalloproteinases and their role in the cytotoxicity. Toxicon 58:277-284.

Leonardi A, Biass D, Kordiš D, Stöcklin R, Favreau P, Križaj I. 2012. Conus consors snail venom proteomics proposes functions, pathways, and novel families involved in its venomic system. Journal of Proteome Research 11:5046-5058.

Lewis Ames C, Ryan JF, Bely AE, Cartwright P, Collins AG. 2016. A new transcriptome and transcriptome profiling of adult and larval tissue in the box jellyfish Alatina alata: an emerging model for studying venom, vision and sex. BMC Genomics 17:650.

Loukas A, Mullin NP, Tetteh KK, Moens L, Maizels RM. 1999. A novel C-type lectin secreted by a tissue-dwelling parasitic nematode. Current Biology: $C B$ 9:825-828.

Love MI, Huber W, Anders S. 2014. Moderated estimation of fold change and dispersion for RNA-seq data with DESeq2. Genome Biology 15:550.

Macrander J, Brugler MR, Daly M. 2015. A RNA-seq approach to identify putative toxins from acrorhagi in aggressive and non-aggressive Anthopleura elegantissima polyps. BMC Genomics 16:221.

Marchler-Bauer A, Derbyshire MK, Gonzales NR, Lu S, Chitsaz F, Geer LY, Geer RC, He J, Gwadz M, Hurwitz DI, Lanczycki CJ, Lu F, Marchler GH, Song JS, Thanki N, Wang Z, Yamashita RA, Zhang D, Zheng C, Bryant SH. 2015. CDD: NCBI's conserved domain database. Nucleic Acids Research 43:D222-6.

Moran Y, Genikhovich G, Gordon D, Wienkoop S, Zenkert C, Ozbek S, Technau U, Gurevitz M. 2012. Neurotoxin localization to ectodermal gland cells uncovers an alternative mechanism of venom delivery in sea anemones. Proceedings. Biological sciences / The Royal Society 279:1351-1358.

PeerJ reviewing PDF | (2021:07:63176:1:1:NEW 15 Oct 2021) 
615 Moran Y, Praher D, Schlesinger A, Ayalon A, Tal Y, Technau U. 2013. Analysis of Soluble 616 Protein Contents from the Nematocysts of a Model Sea Anemone Sheds Light on Venom

617

618

619

620

621

622

623

624

625

626

627

628

629

630

631

632

633

634

635

636

637

638

639

640

641

642

643

644

645

646

647

648

649

650

651

652

653

654

655

656

657

658

659
Evolution. Marine Biotechnology 15:329-339. DOI: 10.1007/s10126-012-9491-y.

Morita T. 2005. Structures and functions of snake venom CLPs (C-type lectin-like proteins) with anticoagulant-, procoagulant-, and platelet-modulating activities. Toxicon 45:1099-1114.

Okamura B, Gruhl A, Bartholomew JL. 2015. An introduction to myxozoan evolution, ecology and development. In: Okamura B, Gruhl A, Bartholomew JL, eds. Myxozoan Evolution, Ecology and Development. Switzerland: Springer International Publishing. 1-20

Orts DJB, Moran Y, Cologna CT, Peigneur S, Madio B, Praher D, Quinton L, De Pauw E, Bicudo JEPW, Tytgat J, de Freitas JC. 2013. BcsTx3 is a founder of a novel sea anemone toxin family of potassium channel blocker. The FEBS journal 280:4839-4852.

Östman C. 2000. A guideline to nematocyst nomenclature and classification, and some notes on the systematic value of nematocysts. Scientia Marina 64:31-46. DOI:

10.3989/scimar.2000.64s131.

Patro R, Duggal G, Love MI, Irizarry RA, Kingsford C. 2017. Salmon provides fast and biasaware quantification of transcript expression. Nature Methods 14:417-419.

Piriatinskiy G, Atkinson SD, Park S, Morgenstern D, Brekhman V, Yossifon G, Bartholomew JL, Lotan T. 2017. Functional and proteomic analysis of Ceratonova shasta (Cnidaria: Myxozoa) polar capsules reveals adaptations to parasitism. Scientific Reports 7:9010.

Podobnik M, Anderluh G. 2017. Pore-forming toxins in Cnidaria. Seminars in Cell \& Developmental Biology 72:133-141.

Rachamim T, Morgenstern D, Aharonovich D, Brekhman V, Lotan T, Sher D. 2015. The Dynamically Evolving Nematocyst Content of an Anthozoan, a Scyphozoan, and a Hydrozoan. Molecular Biology and Evolution 32:740-753. DOI: 10.1093/molbev/msu335.

Ramírez-Carreto S, Vera-Estrella R, Portillo-Bobadilla T, Licea-Navarro A, Bernaldez-Sarabia J, Rudiño-Piñera E, Verleyen JJ, Rodríguez E, Rodríguez-Almazán C. 2019. Transcriptomic and Proteomic Analysis of the Tentacles and Mucus of Anthopleura dowii Verrill, 1869. Marine Drugs 17:436. DOI: 10.3390/md17080436.

Ranasinghe SL, Fischer K, Gobert GN, McManus DP. 2015. Functional expression of a novel Kunitz type protease inhibitor from the human blood fluke Schistosoma mansoni. Parasites \& Vectors 8:408.

Rokyta DR, Wray KP, Margres MJ. 2013. The genesis of an exceptionally lethal venom in the timber rattlesnake (Crotalus horridus) revealed through comparative venom-gland transcriptomics. BMC Genomics 14:394. DOI: 10.1186/1471-2164-14-394.

Sachkova MY, Landau M, Surm JM, Macrander J, Singer SA, Reitzel AM, Moran Y. 2020. Toxin-like neuropeptides in the sea anemone unravel recruitment from the nervous system to venom. Proceedings of the National Academy of Sciences 117:27481-27492.

Shi J, Gilbert GE. 2003. Lactadherin inhibits enzyme complexes of blood coagulation by competing for phospholipid-binding sites. Blood 101:2628-2636. DOI: 10.1182/blood2002-07-1951.

Sintsova O, Gladkikh I, Chausova V, Monastyrnaya M, Anastyuk S, Chernikov O, Yurchenko E, Aminin D, Isaeva M, Leychenko E, Kozlovskaya E. 2018. Peptide fingerprinting of the sea anemone Heteractis magnifica mucus revealed neurotoxins, Kunitz-type proteinase inhibitors and a new $\beta$-defensin $\alpha$-amylase inhibitor. Journal of Proteomics 173:12-21. DOI: 10.1016/j.jprot.2017.11.019.

Peer] reviewing PDF | (2021:07:63176:1:1:NEW 15 Oct 2021) 
660

661

662

663

664

665

666

667

668

669

670

671

672

673

674

675

676

677

678

679

680

681

682

683

684

685

686

687

688

689

690

691

Smith D, Cwiklinski K, Jewhurst H, Tikhonova IG, Dalton JP. 2020. An atypical and functionally diverse family of Kunitz-type cysteine/serine proteinase inhibitors secreted by the helminth parasite Fasciola hepatica. Scientific Reports 10:20657.

Soneson C, Love MI, Robinson MD. 2015. Differential analyses for RNA-seq: transcript-level estimates improve gene-level inferences. F1000Research 4:1521.

Takeuchi F, Sekizuka T, Ogasawara Y, Yokoyama H, Kamikawa R, Inagaki Y, Nozaki T, Sugita-Konishi Y, Ohnishi T, Kuroda M. 2015. The Mitochondrial Genomes of a Myxozoan Genus Kudoa Are Extremely Divergent in Metazoa. PLOS ONE 10:e0132030.

$\mathrm{Tu}$ AT, Hendon RR. 1983. Characterization of lizard venom hyaluronidase and evidence for its action as a spreading factor. Comparative Biochemistry and Physiology. B, Comparative Biochemistry 76:377-383.

Voolstra CR, Li Y, Liew YJ, Baumgarten S, Zoccola D, Flot J-F, Tambutté S, Allemand D, Aranda M. 2017. Comparative analysis of the genomes of Stylophora pistillata and Acropora digitifera provides evidence for extensive differences between species of corals. Scientific Reports 7:17583.

Wang P, Jiang Z, Liu X, Yu K, Wang C, Li H, Zhong L. 2020. PI16 attenuates response to sorafenib and represents a predictive biomarker in hepatocellular carcinoma. Cancer Medicine 9:6972-6983.

Whittington CM, Papenfuss AT, Bansal P, Torres AM, Wong ESW, Deakin JE, Graves T, Alsop A, Schatzkamer K, Kremitzki C, Ponting CP, Temple-Smith P, Warren WC, Kuchel PW, Belov K. 2008. Defensins and the convergent evolution of platypus and reptile venom genes. Genome research 18:986-994. DOI: 10.1101/gr.7149808

Yang W, Feng J, Wang B, Cao Z, Li W, Wu Y, Chen Z. 2014a. BF9, the first functionally characterized snake toxin peptide with Kunitz-type protease and potassium channel inhibiting properties. Journal of Biochemical and Molecular Toxicology 28:76-83.

Yang Y, Xiong J, Zhou Z, Huo F, Miao W, Ran C, Liu Y, Zhang J, Feng J, Wang M, Wang M, Wang L, Yao B. 2014b. The genome of the myxosporean Thelohanellus kitauei shows adaptations to nutrient acquisition within its fish host. Genome Biology and Evolution 6:3182-3198.

Zhang M, Fishman Y, Sher D, Zlotkin E. 2003. Hydralysin, a novel animal group-selective paralytic and cytolytic protein from a noncnidocystic origin in hydra. Biochemistry 42:8939-8944.

PeerJ reviewing PDF | (2021:07:63176:1:1:NEW 15 Oct 2021) 


\section{Table 1 (on next page)}

Putative venom-like compounds identified in Ceratonova shasta datasets.

In the "Dataset" column, "P" indicates the compound was identified in the $C$. shasta nematocyst proteome; " $T$ " indicates the transcriptome. Full amino acid sequences of these compounds are provided in Supplemental File 4. 


\begin{tabular}{|c|c|c|c|c|c|}
\hline VLC name & Dataset & Length (aa) & Closest NCBI homolog & $\begin{array}{l}\text { \% Sequence } \\
\text { identity }\end{array}$ & E-value \\
\hline C. shasta lactadherin-like protein & $\mathrm{P}$ & 671 & $\begin{array}{l}\text { Cnidaria: Myxozoa } \\
\text { Myxobolus squamalis } \\
\text { KAF1744687 }\end{array}$ & 45.9 & $2 \mathrm{e}-75$ \\
\hline $\begin{array}{l}\text { C. shasta peptidase inhibitor } 16- \\
\text { like protein }\end{array}$ & $\mathrm{P}, \mathrm{T}$ & 825 & $\begin{array}{l}\text { Cnidaria: Myxozoa } \\
\text { Thelohanellus kitauei } \\
\text { KII69682 }\end{array}$ & 45.8 & $4 e-56$ \\
\hline $\begin{array}{l}\text { C. shasta C-type lectin-like } \\
\text { protein }\end{array}$ & $\mathrm{P}, \mathrm{T}$ & 445 & $\begin{array}{l}\text { Cnidaria: Hydrozoa } \\
\text { Hydra vulgaris } \\
\text { XM_012708536 }\end{array}$ & 29.5 & $1 \mathrm{e}-41$ \\
\hline $\begin{array}{l}\text { C. shasta hyaluronidase-like } \\
\text { transcript }\end{array}$ & $\mathrm{T}$ & 351 & $\begin{array}{l}\text { Chordata: Aves } \\
\text { Rhinopomastus } \\
\text { cyanomelas } \\
\text { NXO00254 }\end{array}$ & 28.4 & $7 e-43$ \\
\hline $\begin{array}{l}\text { C. shasta Kunitz-type protease } \\
\text { inhibitor-like transcript } 1\end{array}$ & $\mathrm{~T}$ & 119 & $\begin{array}{l}\text { Cnidaria: Anthozoa } \\
\text { Actinia tenebrosa } \\
\text { XP_031550075 }\end{array}$ & 47.8 & $6 e-31$ \\
\hline $\begin{array}{l}\text { C. shasta Kunitz-type protease } \\
\text { inhibitor-like transcript } 2\end{array}$ & $\mathrm{~T}$ & 270 & None & & \\
\hline $\begin{array}{l}\text { C. shasta reprolysin-like } \\
\text { transcript }\end{array}$ & $\mathrm{T}$ & 193 & $\begin{array}{l}\text { Chordata: Actinopterygii } \\
\text { Silurus meridionalis } \\
\text { KAF7705091 }\end{array}$ & 28.9 & $7 e-23$ \\
\hline C. shasta astacin-like transcript & $\mathrm{T}$ & 198 & $\begin{array}{l}\text { Cnidaria: Myxozoa } \\
\text { Thelohanellus kitauei } \\
\text { KII65609 }\end{array}$ & 37.6 & $4 e-33$ \\
\hline
\end{tabular}




\section{Figure 1}

Bioinformatic pipeline for identification and characterization of genes coding for venomlike compounds.
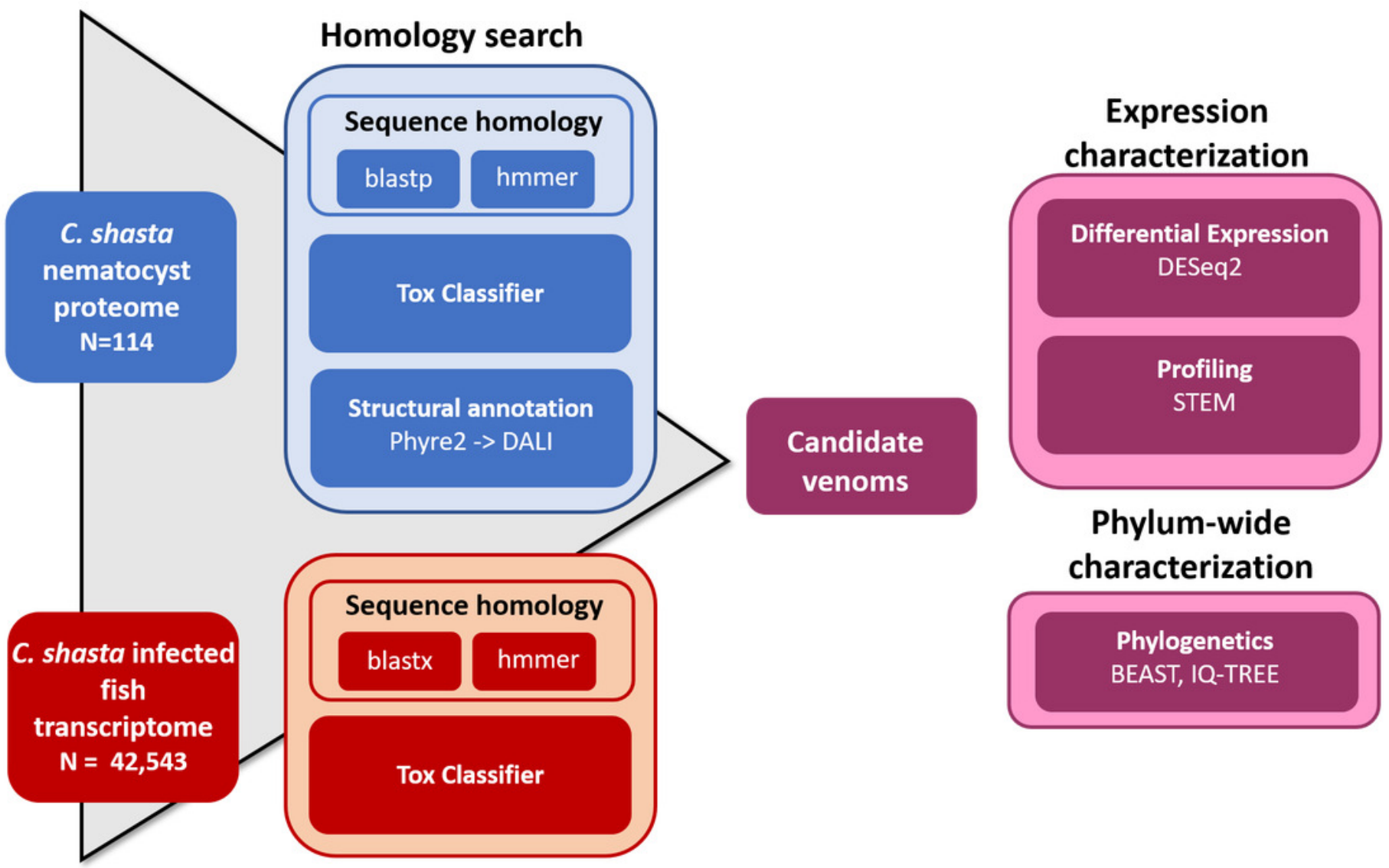
Figure 2

Venn diagrams showing candidate venom-like proteins identified by our screening methods.

A: proteins from the Ceratonova shasta nematocyst. B: Transcripts from C. shasta infection transcriptomes.

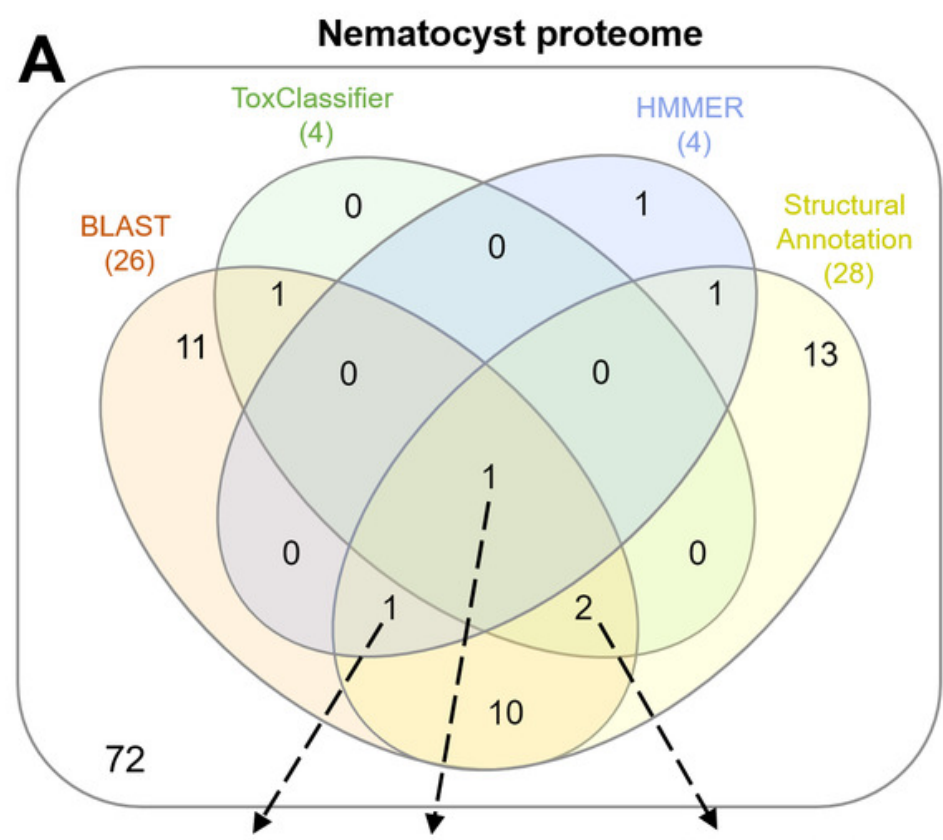

(1) C-type lectin
(1) Peptidase inhibitor 16
(2) Lactadherin

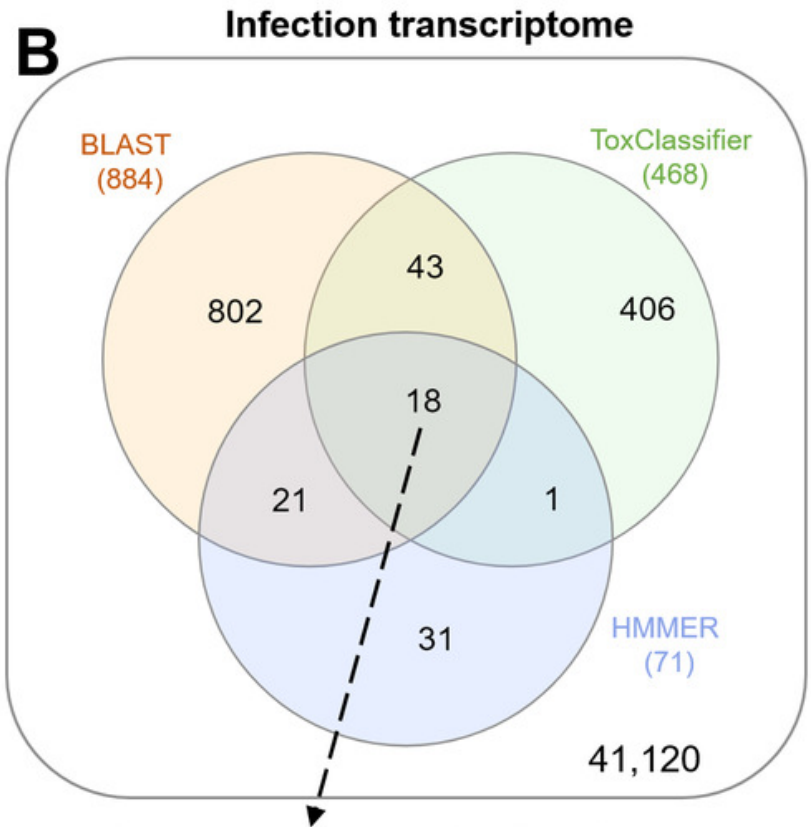

(1) C-type Lectin

(2) Hyaluronidase

(3) Kunitz-type protein

(5) Kunitz-type protein
(1) Metallopeptidase

(4) Metallopeptidase

(2) Peptidase inhibitor 16 


\section{Figure 3}

Results of HMM search of Ceratonova shasta nematocyst proteome and translated $C$. shasta transcriptome from infected fish, against 22 cnidarian venom HMMs from Klompen et al. (2020).

The HMMs in the outer gray box were not identified in C. shasta.

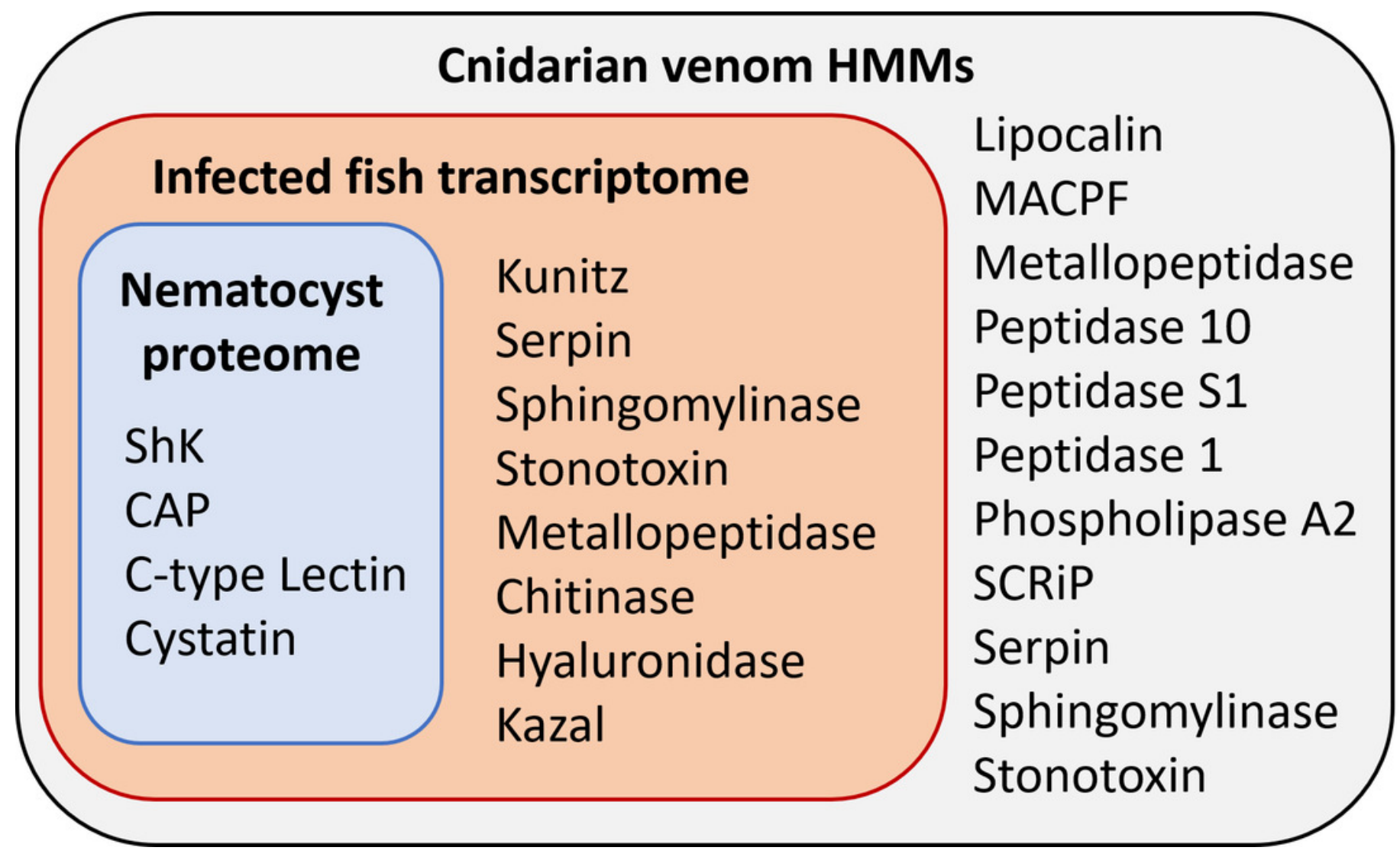


Figure 4

Maximum likelihood phylogenetic trees and protein alignments from two VLCs with domains shown as colored blocks.

A: tree of C-type lectin-like protein has topology in agreement with established cnidarian taxonomy. B: tree of astacin-like transcript has topology incongruent with established cnidarian taxonomy and includes sequences from venomous animals outside of Cnidaria. See Additional File 3 for trees of all candidate VLCs.

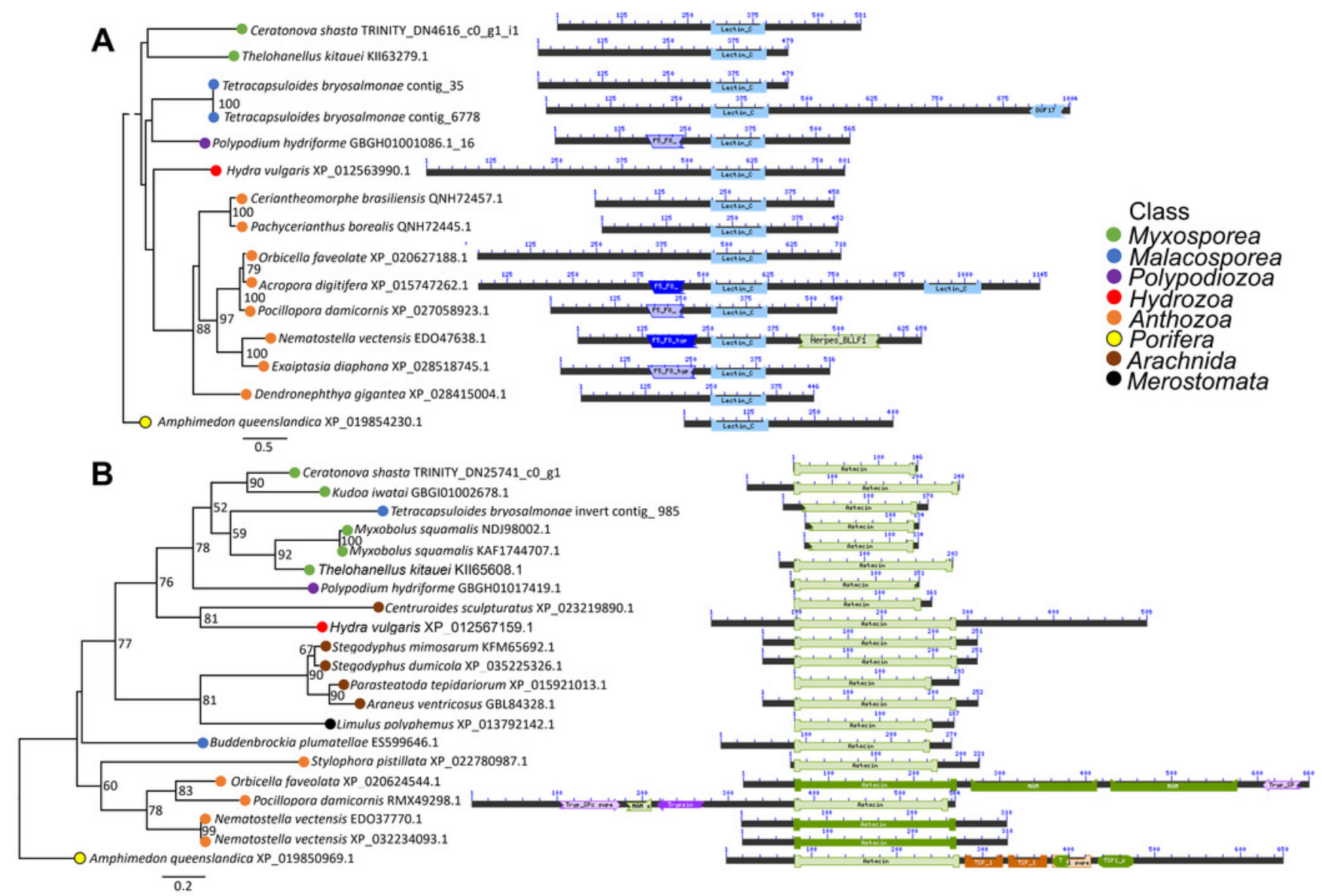




\section{Figure 5}

Heatmap of time-series expression of genes coding for the 8 VLCs, at 1, 7, 14 and 21days post exposure (dpe).

There are three replicate fish per timepoint, each represented by a square that is shaded by gene read count, normalized for parasite replication. STEM Profiles are assigned to sequences by expression. Profiles with the same color belong to the same expression cluster.

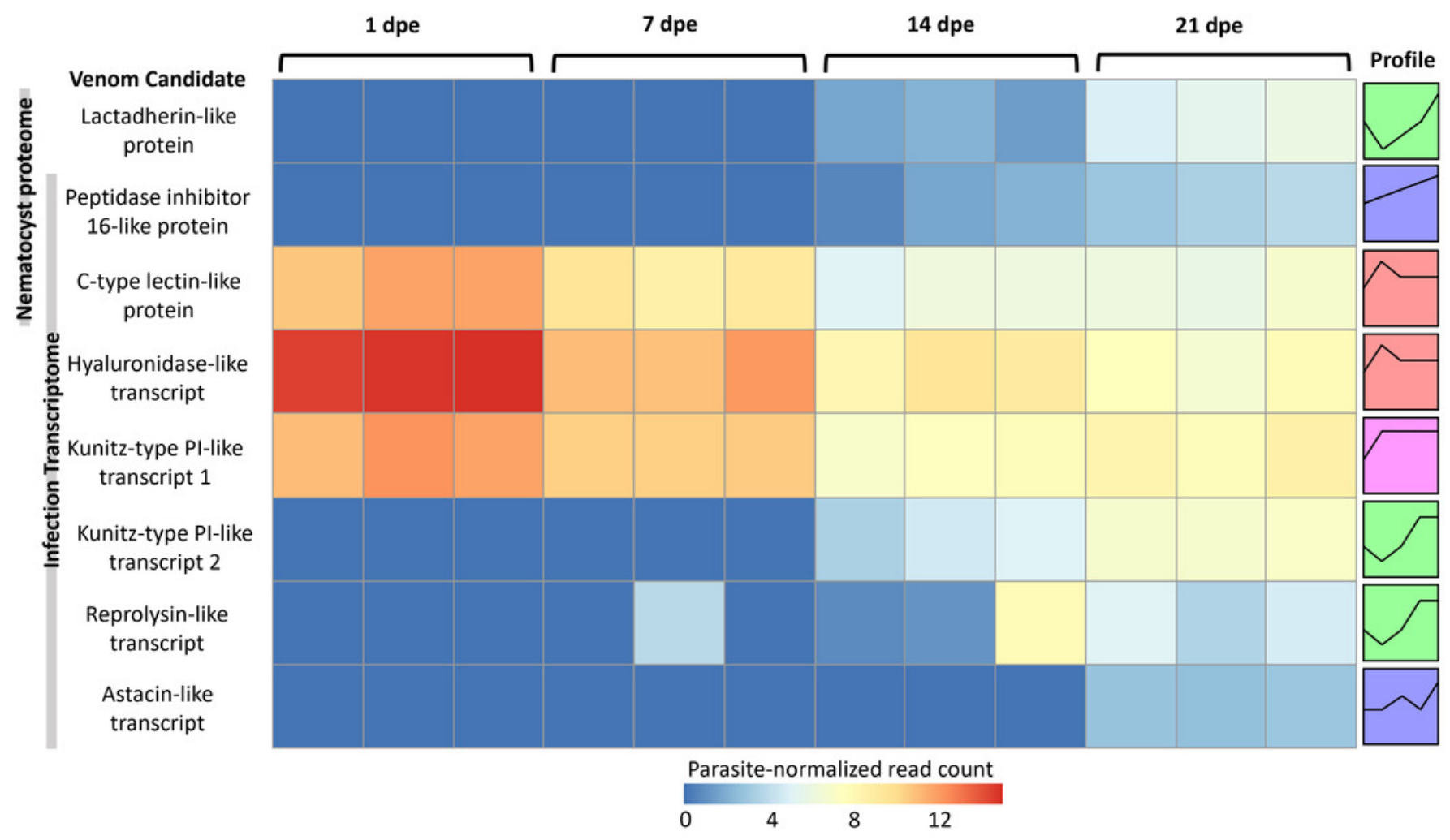

